\title{
Uma Proposta de Camada de Aplicação baseada no Padrão IEEE 11073 para Simulação de Aplicações de Saúde Digital em Redes Corporais sem Fio
}

\author{
Robson A. Lima, Vinicius C. Ferreira, Egberto Caballero, \\ Célio V. N. Albuquerque, Débora C. Muchaluat Saade \\ ${ }^{1}$ Laboratório MídiaCom, Instituto de Computação - Universidade Federal Fluminense (UFF) \\ Niterói - RJ - Brasil \\ \{robsonal, vinicius, egbertocr, celio, debora\}@midiacom.uff.br
}

\begin{abstract}
Simulating new protocols in WirelessBody Area Networks (WBANs) is important to evaluate new proposals before developing a real implementation. However, WBAN simulators only provide generic network applications, thus it is not possible to simulate a health device in a WBAN network. Therefore, this work proposes an application layer based on the ISO/IEEE 11073 Standard (X73-PHD) for simulating e-health applications in WBANs. Our proposal is implemented for the Castalia simulator, using the Antidote library, an open implementation of X73-PHD. Simulation results show that the X73-PHD standard communication model does not perform well due to long timeouts defined in the standard. Therefore, this paper also proposes a new communication mode, which reduces the exchange of control packets and the number of associations made by personal health devices in WBANs.
\end{abstract}

Resumo. Simular novos protocolos em Redes Corporais sem Fio (Wireless Body Area Networks - WBANs) é importante para fazer uma avaliação da proposta antes de partir para o desenvolvimento de uma implementação real. Contudo, simuladores de WBANs apenas oferecem aplicações genéricas de rede, não sendo possível simular um dispositivo de saúde em uma rede WBAN. Sendo assim, este trabalho propõe uma camada de aplicação baseada no Padrão ISO/IEEE 11073 para simulação de aplicações de saúde digital em WBANs. A proposta é implementada no simulador Castalia, usando como base a biblioteca Antidote, uma implementação de código aberto de dispositivos ISO/IEEE 11073 (X73-PHD). Resultados de simulação mostram que o modelo de comunicação padrão da norma X73-PHD não apresenta um bom desempenho devido a longos timeouts definidos na norma. Portanto, este artigo também propõe um novo modo de comunicação, que reduz a troca de pacotes de controles e o número de associações feitas pelos dispositivos de saúde em WBANs.

\section{Introdução}

Redes de Sensores Sem Fio (RSSF) têm se tornado um ramo de estudo importante para inúmeros cenários de aplicação e simulação (internet das coisas, cidades inteligentes,

Este trabalho foi parcialmente financiado pelo CNPq, CAPES, FAPERJ, FAPESP e INCT-MACC. 
informática médica, etc). Devido ao aumento de pesquisas na área de RSSF, juntamente com telemedicina, um novo tipo de rede surgiu: as redes corporais sem fio ou Wireless Body Area Networks (WBANs) [Movassaghi et al. 2014]. Uma WBAN é formada por dispositivos inteligentes colocados sobre pele ou implantados no corpo, que são capazes de trocar dados através de uma rede sem fio [Ferreira et al. 2017].

A ausência de dispositivos pessoais de saúde no mercado e os riscos que testes práticos podem oferecer à saúde tornam experimentos reais com WBANs complexos. Com o avanço e aperfeiçoamento de RSSF, simuladores se tornaram uma importante ferramenta que permite viabilizar testes com menor custo e tempo. O Castalia [Boulis, Athanassios 2013] é um simulador de redes de sensores sem fio, sensores corporais e dispositivos embutidos de baixa potência. Ele é um software livre e de código aberto, desenvolvido para que os usuários testem seus próprios algoritmos e protocolos.

O Castalia fornece uma estrutura genérica, confiável e realista para a primeira validação de um algoritmo, antes de passar para uma plataforma de sensor específica. Cada sensor é representado por um nó genérico, que executa funções de transmissão e recepção de dados. Entretanto, as aplicações disponíveis para testes de novos protocolos no simulador são genéricas, isto é, não especificam o tipo de sensor nem seus requisitos e procedimentos de comunicação na camada de aplicação. Isso representa um ponto fraco para o uso do simulador para aplicações de saúde digital, já que os protocolos de aplicação padronizados não são considerados nas simulações.

Para tornar as simulações mais realistas, o uso de aplicações reais e padronizadas é um ponto vital. O padrão ISO/IEEE 11073 Personal Health Devices (X73-PHD) descreve aplicações e informações para dispositivos de telessaúde. O padrão também especifica a troca e a representação de dados para comunicação entre dispositivos pessoais de saúde. Sendo assim, este artigo propõe o uso do padrão ISO/IEEE 11073 para simulação de aplicações de saúde digital em cenários de WBAN. Desta forma, simulações de novos protocolos em WBANs se tornam mais realistas com o uso de uma camada de aplicação baseada no padrão ISO/IEEE 11073.

O termo PHD envolve tanto dispositivos médicos quanto dispositivos para exercícios físicos usados pelos próprios usuários em suas casas [ISO/IEEE 2012a]. A família de padrões ISO/IEEE 11073 é divida em três grupos, a primeira e mais antiga parte é a ISO/IEEE 11073 Lower Layer, que especifica protocolos e serviços de comunicação orientados à conexão, utilizando camadas físicas como infravermelho, tecnologia RF sem fio ou Ethernet [ISO/IEEE 2004]. Já a parte ISO/IEEE 11073 Point-of-Care-Devices especifica padrões de comunicação para dispositivos que são usados exclusivamente em unidades de saúde. Por fim, a ISO/IEEE 11073 Personal Health Devices (PHD), ou Dispositivo Pessoal de Saúde (DPS), define padrões para dispositivos usados pelos usuários em suas casas. O foco deste trabalho será apenas no padrão ISO/IEEE 11073 Personal Health Devices, que especifica dispositivos usados mais frequentemente por usuários comuns, tais como pacientes em suas casas ou atletas que precisam ter sua atividade física monitorada, representando uma parte significativa das aplicações de saúde digital. Por questões de simplificação, este padrão será referenciada apenas como X73-PHD neste texto.

O padrão X73-PHD define dois tipos de dispositivos: agentes e gerentes. Os 
agentes são tipicamente sensores ou atuadores, de baixa potência e com pouco poder de processamento, enquanto os gerentes são dispositivos com um poder de processamento maior, que podem ou não estar conectados a uma fonte de energia. Apesar do padrão X73-PHD não comentar explicitamente, dispositivos agentes e gerentes poderiam ser nós de uma WBAN. Existem dois modos de comunicação no padrão X73-PHD, o modo com confirmação e sem confirmação. No modo sem confirmação, as leituras são transmitidas sem nenhum tipo de confirmação de recebimento por parte do gerente. O modo com confirmação espera que, para cada pacote de leitura enviado pelos agentes, um acknowledgement (ACK) seja transmitido, reconhecendo que o pacote foi entregue no gerente. De acordo com o padrão, caso o agente não receba o ACK num período de $3 \mathrm{~s}$, uma nova associação com o gerente deve ser feita para finalizar a transmissão de leituras.

Este último modo de comunicação pode aumentar consideravelmente o número de pacotes de controle trocados entre agente e gerente em um cenário WBAN, quando um ACK é perdido. Para evitar esse overhead, este artigo também propõe um novo modo de comunicação, chamado modo de retransmissão. Este modo é baseado em stop-andwait, que retransmite o pacote perdido, ao invés de solicitar imediatamente uma nova associação.

O restante do artigo está estruturado da seguinte forma. Na Seção 2, apresentamse trabalhos relacionados sobre o padrão X73-PHD. A Seção 3 apresenta a proposta deste artigo, detalhando o novo modo de comunicação proposto como uma extensão ao padrão X73-PHD. Resultados de simulações com o Castalia são discutidos na Seção 4. Por fim, as considerações finais e trabalhos futuros são comentados na Seção 5.

\section{Trabalhos Relacionados}

A parte IEEE 11073-20601 Optimized Exchange Protocol é o principal componente da família X73-PHD. Ele define a sintaxe de comunicação usando um Modelo de Domínio de Informação (Domain Information Model DIM), máquinas de estados e tipos de serviços de comunicação no Modelo de Serviço e procedimentos no Modelo de Comunicação

A família de padrões 11073 oferece perfis de especialização, isto é, cada agente possui suas próprias normas, as quais descrevem sua representação de dados, atributos, métodos e eventos. Por exemplo, o perfil IEEE 11073-10408 define as classes e objetos para um termômetro, já o perfil IEEE 11073-10415 faz o mesmo para uma balança.

A biblioteca Antidote é uma implementação da parte IEEE 11073-20601 Optimized Exchange Protocol, desenvolvida pela empresa Signove, como parte da plataforma SigHealth ${ }^{1}$. Esta biblioteca é a primeira implementação livre e de código aberto deste padrão, e foi desenvolvida em ANSI-C com arquitetura modular, o qual permite portabilidade de código para diferentes plataformas.

Com a popularização da família de padrões 11073, vários aperfeiçoamentos e integrações com outros sistemas foram feitos. Nos trabalhos [Martins et al. 2014], [Gomes et al. 2015] e [Santos et al. 2014], é proposta a integração do padrão X73-PHD com protocolos de IoT, como MQTT e CoAP, para serem usados na camada de transporte de PHDs. Esses protocolos auxiliariam o compartilhamento de informação de saúde di-

\footnotetext{
${ }^{1}$ SigHealth é uma plataforma de monitoramento remoto de pacientes e gestão de dados utilizando dispositivos pessoais sem fio para a saúde.
} 
retamente na Internet, usando baixo consumo energético e poucos pacotes de controle. Nesse trabalho, também é apresentado o mapeamento das mensagens X73-PHD para mensagens MQTT e CoAP e a biblioteca Antidote é utilizada na camada de aplicação.

O padrão X73-PHD é aplicada em várias áreas da saúde e de e-Health, como por exemplo, diagnóstico precoce de doenças crônicas e monitoramento remoto de pacientes em suas residências. No trabalho [Jianchu and Steve 2005], os autores propõem uma coleção de dispositivos vestíveis X73-PHD, plug-and-play, que coletam continuamente, dados fisiológicos e do ambiente. Neste projeto, três dispositivos são utilizado: dispositivos sensores, que coletam informações fisiológicas e do ambiente; Os Data Loggers, que são dispositivos móveis, que armazenam temporariamente os dados dos sensores; e por fim, as Base Stations, terminais que recebem, processam, armazenam e exibem informações recebidas do data loggers. Como camada de transporte os autores, utilizaram o Bluetooth e, na camada de aplicação, o padrão X73-PHD.

Outro trabalho interessante é o desenvolvimento de um adaptador para dispositivos que não seguem o padrão X73-PHD. Como mostrado em [Korsanov et al. 2013], os autores sugerem um dispositivo intermediário entre os sensores não padronizados e o gerente. Este dispositivo faz a tradução das mensagens vindo desses sensores para mensagens X73-PHD, e então transmite os dados traduzidos para um gerente padronizado. Este trabalho não deixa claro como foram feitos o mapeamento das mensagens e em quais dispositivos o adaptador proposto funciona.

Neste artigo, usamos a biblioteca Antidote para propor uma camada de aplicação, seguindo o padrão X73-PHD no simulador Castalia. Cinco perfis de especialização diferentes são utilizados: 11073-10406 electrocardiograma básico de 3 derivações, 1107310404 oxímetro de pulso, 11073-10408 termômetro, 11073-10417 medidor de glicose e 11073-10407 medidor de pressão sanguínea. Além disso, um novo modo de comunicação é proposto como extensão ao padrão para uso em WBANs, como será detalhado na próxima seção.

\section{Simulação de Aplicações de Saúde Digital em WBANs}

Este trabalho propõe o uso do padrão ISO/IEEE 11073 para simulação de aplicações de saúde digital em cenários de WBAN. Conforme o padrão, são propostos um componente gerente e outro agente na Camada de Aplicação do Simulador Castalia. O envio de dados pode ocorrer nos dois sentidos, do gerente para o agente ou do agente para o gerente. Entretanto, neste trabalho, o agente sempre inicia os envios de leituras para o gerente, simulando o envio de leituras de sensores ao nó concentrador da WBAN. O agente também é o primeiro e o único a enviar a mensagem de Association request para solicitar uma associação ao gerente antes de iniciar o envio de leituras. Quando não há mais leituras para serem enviadas, o agente envia a mensagem Association release para encerrar a associação.

A implementação atual oferece cinco dispositivos pessoais de saúde como agentes, um oxímetro de pulso, um medidor de glicose, um termômetro, um medidor de pressão e um eletrocardiograma (ECG de 3 derivações). O oxímetro transmite a frequência cardíaca em batimentos por minuto e a percentagem de saturação de oxigênio na hemoglobina arterial $\left(\mathrm{SpO}_{2}\right)$. O medidor de glicose transmite o nível de glicose, isto é, a concentração de açúcar no sangue em miligramas por decilitro (mg/dL). O termômetro 
transmite a temperatura em Celsius $\left({ }^{\circ} \mathrm{C}\right)$. $\mathrm{O}$ medidor de pressão envia uma mensagem composta por três medidas arteriais, sistólica, diastólica e a média arterial em milímetros de mercúrio (mmHg). O ECG envia oitenta amostras do potencial elétrico do coração em milivolts $(\mathrm{mV})$ por pacote. Todos os dados dos agentes são produzidos de forma pseudoaleatória, exceto o ECG que transmite valores reais obtidos a partir da base de dados [Goldberger et al. 2000].

O padrão X73-PHD define eventos com confirmação e sem confirmação. Os eventos com confirmação esperam uma mensagem de reconhecimento do gerente enquanto os eventos sem confirmação não. Mensagens de controle como Association request e Association release são sempre eventos que esperam uma confirmação, porém o envio de leituras pode ser ou não confirmado.

Se um agente usa o modo de comunicação confirmado e o ACK de uma leitura enviada ao gerente não é recebido, o padrão indica que o agente deve realizar uma nova associação com o gerente para transmitir novamente a leitura. Em um cenário de rede sem fio, a perda de quadros pode ocorrer com frequência, por isso, o modo de comunicação confirmado proposto no padrão pode levar a uma sobrecarga de pacotes de controle com necessidade de estabelecer uma nova associação toda vez que um ACK for perdido na WBAN.

Para contornar este problema, foi utilizado o novo modo de comunicação proposto, chamado de modo de retransmissão, como extensão ao padrão ISO/IEEE 11073. As próximas subseções detalham os três modos de operação e discutem como a proposta deste artigo pode melhorar o funcionamento de Personal Health Devices em cenários WBANs.

\subsection{Eventos sem confirmação}

A Figura 1 mostra um diagrama de sequência do procedimento de troca de mensagens de uma operação normal de um agente com configuração padrão e usando eventos de envio de leituras sem confirmação. O agente pretende se associar com o gerente pela primeira vez e então envia um Association request. Quando o gerente recebe o Association request, ele verifica se já houve associações feitas previamente com esse agente. Se for a primeira associação, o gerente envia uma mensagem de Get attributes juntamente com uma mensagem de Association response. Logo após, o agente envia sua configuração e atributos para iniciar a transmissão de leituras. Quando todas as leituras tiverem sido enviadas, o agente envia uma mensagem de Association release e o gerente responde com uma mensagem Association release response para confirmar o término desta associação.

\subsection{Eventos com confirmação}

A Figura 2 descreve o diagrama de sequência da operação de um agente com configuração padrão usando eventos de envio de leitura com confirmação. O processo inicial é idêntico ao explicado na Seção 3.1. A diferença é que para cada leitura que o agente envia, o gerente confirma com uma mensagem ACK. Após enviar uma leitura, o agente espera a confirmação por três segundos, se nada chegar durante esse período, o agente envia uma mensagem de interrupção, Association abort, e transita para o estado de máquina não associado. Se ainda existirem leituras para serem enviadas, o agente deve tentar uma nova associação. 


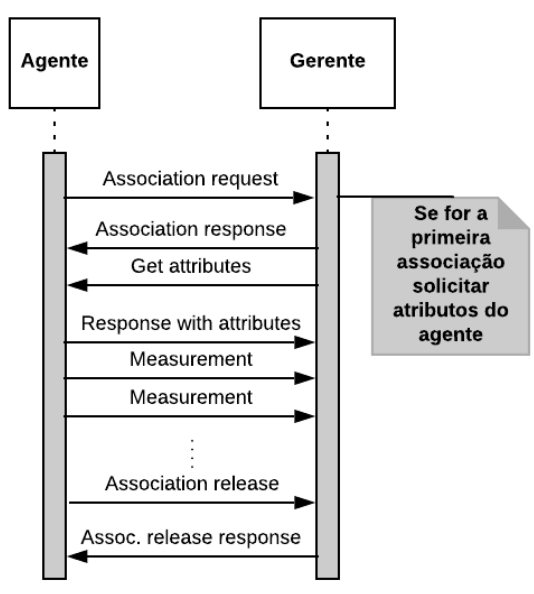

Figura 1. Diagrama de sequência para o modo de operação sem confirmação de uma aplicação X73-PHD.

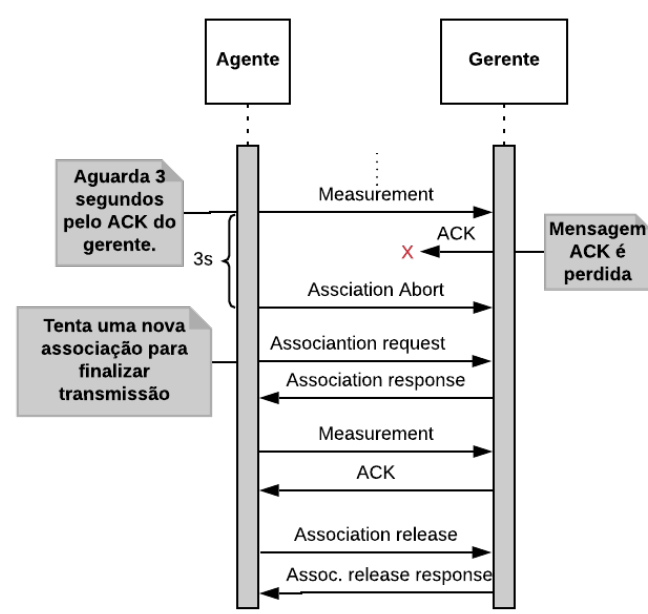

Figura 2. Diagrama de sequência para o modo de operação com confirmação de uma aplicação X73-PHD.

\subsection{Proposta de Modo de Retransmissão}

O padrão X73-PHD assume que, em dispositivos reais, haverá um camada de transporte confiável disponível para comunicação, como o Bluetooth Health Device Profile. Em uma WBAN, pode não haver camada de transporte confiável disponível, já que os sensores têm poucos recursos de memória, processamento e bateria. Desta forma, este artigo propõe um novo modo de comunicação, baseado em stop-and-wait, para realizar retransmissões de pacotes perdidos ou não confirmados pelo gerente.

Isso reduz a troca desnecessária de vários pacotes de controle no processo de associação. Ao invés de fazer uma nova associação quando um ACK não é recebido, retransmite-se o pacote até que um ACK seja recebido. O usuário define o número $n$ de retransmissões, e, então, aquela leitura será retransmitida até $n$ vezes ou até que um ACK seja recebido. Se o gerente receber uma mensagem duplicada, ele retransmitirá imediatamente o ACK que não foi recebido pelo agente, ou seja, o último ACK enviado.

$\mathrm{Na}$ fase de associação, a implementação reduz o timeout de 10 para 0.4 segundos. Após a associação, o dispositivo usando o o Modo de Retransmissão, envia sua primeira leitura, aguarda o período de timeOutToRetransmitPacket conforme definido pelo usuário no arquivo de configuração da simulação. Se nenhuma resposta for recebida, o pacote é retransmitido no máximo maxNumOfRetransmition vezes, conforme definido também pelo usuário, ou até que uma mensagem de reconhecimento do gerente seja recebida. $\mathrm{Na}$ Figura 3 é apresentado o diagrama de sequência para os casos onde uma mensagem ACK e uma leituras são perdidos. Se todas as tentativas definidas em maxNumOfRetransmition forem utilizadas, então uma nova associação é feita com o gerente e a variável maxNumOfRetransmition é redefinida para 0 . Esse processo continua até o agente realizar 3 associações, conforme definido no padrão X73-PHD. Na quarta tentativa, o agente envia uma mensagem de Association abort e volta para estado de máquina não associado.

$\mathrm{Na}$ Seção 4, é avaliada a eficiência desta proposta usando como métrica a redução no envio de pacotes de controle, o número de novas associações feitas por cada dispo- 
sitivo, a quantidade de pacotes retransmitidos e a número de leituras transmitidas com sucesso para o gerente. Vale ressaltar que esta é uma nova proposta e não está presente no padrão X73-PHD.

A implementação no simulador Castalia foi realizada utilizando a biblioteca Antidote [Signove 2011] como base. Ao usar a nova camada de aplicação disponível, os usuários também podem definir alguns parâmetros de simulação, que são: o tipo de dispositivo a ser simulado (termômetro, oxímetro de pulso, medidor de pressão sanguínea, medidor de glicose ou ECG), a taxa de transmissão de leituras por segundo, o modo de operação, com/sem confirmação ou optar em usar a proposta deste artigo, o modo de retransmissão. Neste último modo, o número de tentativas de retransmissão e os timeouts também podem ser configurados.

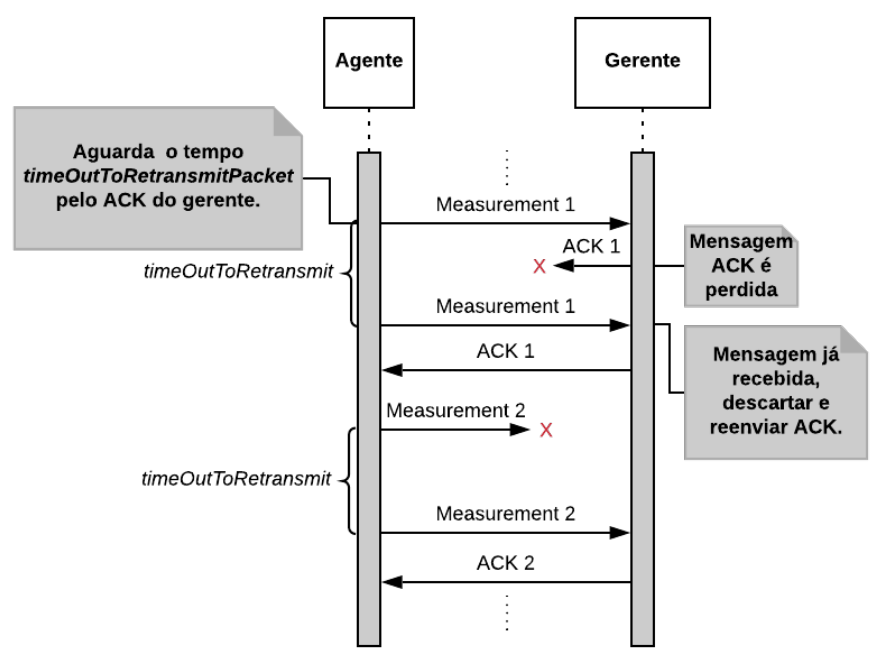

Figura 3. Diagrama de sequência do modo de retransmissão demonstrando o comportamento quando uma mensagem ACK e uma leitura são perdidas.

\section{Resultados}

Todos os resultados apresentados nesta seção são relativos à camada de aplicação implementada por este trabalho. As simulações foram feitas em um computador com 8GB de mémoria RAM, Intel Core i5-7200U e sistema operacional Ubuntu 18.04.1 LTS. A simulação tem duração de $101 \mathrm{~s}$, sendo o primeiro segundo apenas para a configuração da camada MAC. Foram executadas 15 repetições da simulação com intervalo de confiança de $95 \%$.

\subsection{Casos de Uso e Parâmetros da Simulação}

Monitoramento remoto de pacientes e independência para a terceira idade são casos de uso discutidos no padrão X73-PHD. Os sensores e atuadores propostos para esses dois casos de usos são: medidor de pressão, termômetro, medidor de glicose, oxímetro de pulso e um eletrocardiograma.

A Figura 4 mostra a topologia utilizada nas simulações. O hub, que é o nó gerente, está localizado no quadril direito, um nó sensor em cada pulso e um em cada tornozelo e um sensor no meio do peito. Utilizando essas posições, temos a vantagem de utilizar um canal sem fio que já possui um modelo de perda de sinal, entre 
cada par de dispositivos, pré-definido com experimentos reais, conforme apresentado em [Boulis and Tselishchev 2010].

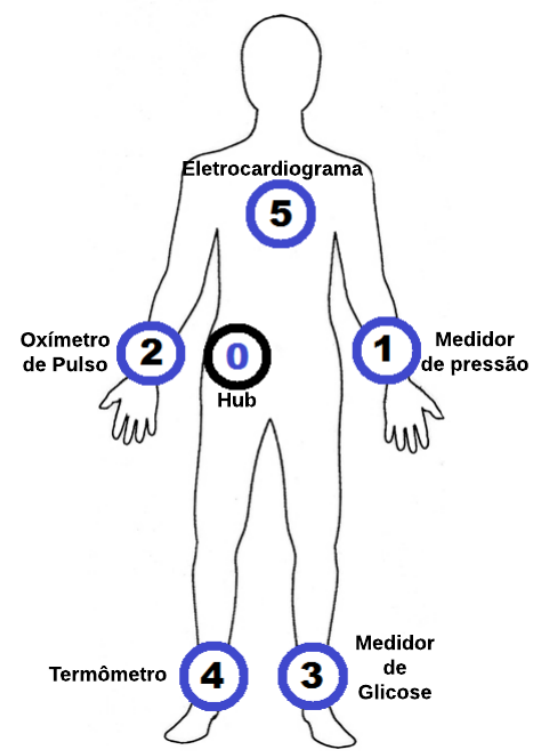

Figura 4. Topologia da rede utilizada nas simulações.

O cenário de simulação utilizado foi definido da seguinte forma: utilizando o modo de retransmissão, os agentes esperam por uma confirmação dos pacotes enviados, por um tempo definido pelo usuário no parâmetro timeOutToRetransmitPacket, se nenhuma confirmação é recebida, é feita a retransmissão desse pacote por até maxNumOfRetransmition vezes, também definido pelo usuário. Se o valor maxNumOfRetransmition de retransmissões for atingido, uma nova associação é estabelecida. Foram usados cinco valores diferentes para timeOutToRetransmitPacket, 200, 400, 600, 800 e 1000 milissegundos. Para maxNumOfRetransmition utilizamos 6, 7, 8, 9 e 10, totalizando 25 cenários diferentes.

Foi utilizada a camada MAC definida pelo padrão IEEE 802.15.6 (WBAN) [ISO/IEEE 2012b] com mapa de perda de sinal e modelo temporal para o canal sem fio fornecidos pelo simulador Castalia. A taxa de transmissão na camada física de $1024 \mathrm{Kbps}$ e o rádio utilizado, se enquadram na proposta do padrão IEEE 802.15.6, trabalhando com $-15 \mathrm{dBm}$ para transmissão.

As configurações dos nós são definidas da seguinte forma: O nó zero é o hub e usa a aplicação Manager. Os demais nós são agentes e usam a aplicação Agent. Ambas as aplicações foram desenvolvidas por este trabalho. O medidor de pressão e o oxímetro de pulso transmitem uma leitura por segundo para o gerente, totalizando 100 leituras durante toda a simulação. O termômetro envia uma leitura a cada 2 segundos, assim, 50 leituras devem ser enviadas durante a simulação. O medidor de glicose transmite apenas uma leitura a cada 25 segundos, totalizando no final da simulação 4 leituras a serem enviadas. Neste trabalho, é assumido que o ECG é um dispositivo que recebe os sinais de todos os eletrodos que estiverem implantados no corpo e envia os sinais para o gerente. Este ECG transmitirá 80 leituras por pacote a cada 0.8 segundos, o que no final totaliza 125 leituras. Essas 80 amostras equivalem a 0.8 segundos de dados retirados da base de dados 
[Goldberger et al. 2000].

\subsection{Análise dos resultados}

Analisar a quantidade de leituras que foram entregues com sucesso no gerente é fundamental para averiguar a eficiência do método de transmissão. Na Figura 5, é exibida a quantidade de leituras entregues por cada um dos dispositivos, em cada um dos vinte e cinco cenários analisados, utilizando o mecanismo proposto e, na Figura 6 é possível ver o desempenho dos três modos onde, foi utilizado o cenário maxNumOfRetransmition $=6$ e timeOutToRetransmitPacket $=200 \mathrm{~ms}$ para o modo de retransmissão.

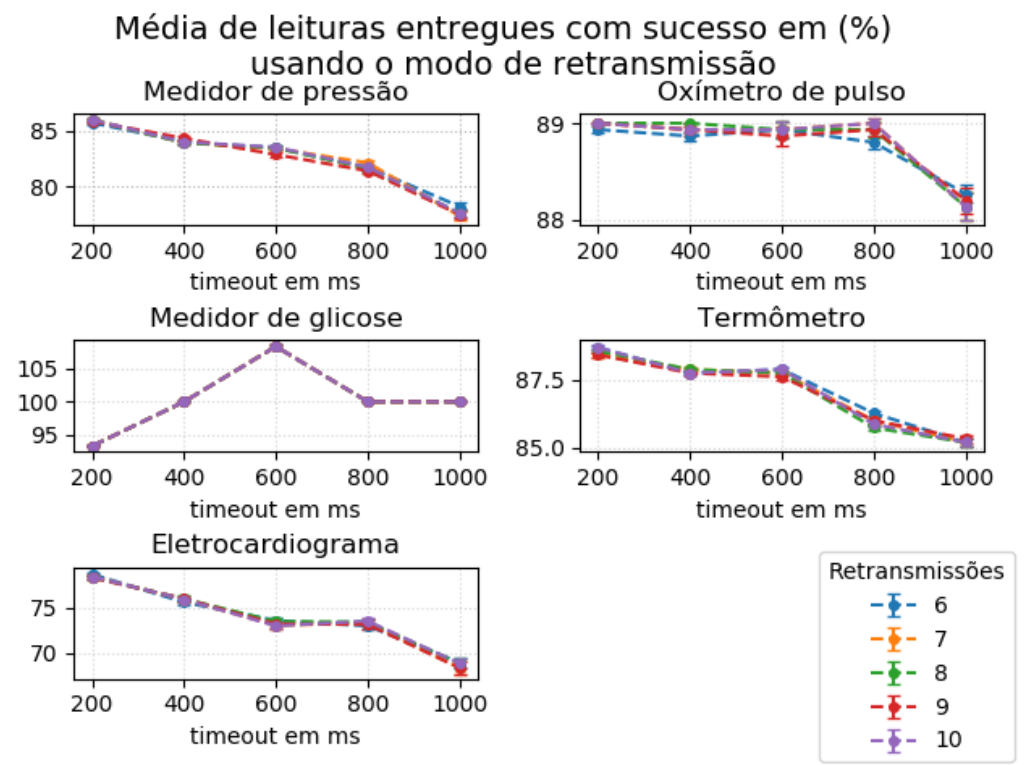

Figura 5. O eixo $y$ representa a percentagem de leituras entregues e o eixo $x$ os valores de timeOutToRetransmitPacket. Cada linha do gráfico representa um valor de maxNumOfRetransmition.

É possível observar na Figura 5 que a maioria dos pacotes são transmitidos quando o menor timeout de 200 milissegundos é utilizado. Este resultado é esperado, pois a simulação possui tempo finito e, quanto maior o timeout, menos tempo para enviar leituras o dispositivo possui. Por outro lado, a qualidade do enlace sem fio e a quantidade de leituras transmitidas influenciam diretamente neste resultado. Enquanto o medidor de glicose envia apenas quatro leituras em 100 s, o ECG - que possui o pior link entre todos os dispositivos, deve enviar $125 \mathrm{em} 100 \mathrm{~s}$ apenas.

Quando todas as tentativas de retransmissão são utilizadas e, nenhuma resposta é recebida, o dispositivo tenta uma nova associação para finalizar a transmissão dos pacotes remanescentes ou não confirmados. Após a nova associação, as leituras são transmitidas com um novo número de sequência, o que pode causar o recebimento de pacotes desnecessários no gerente. Isso pode ser visto na Figura 5, onde a percentagem de leituras recebidas com sucesso do medidor de glicose atinge $105 \%$. 


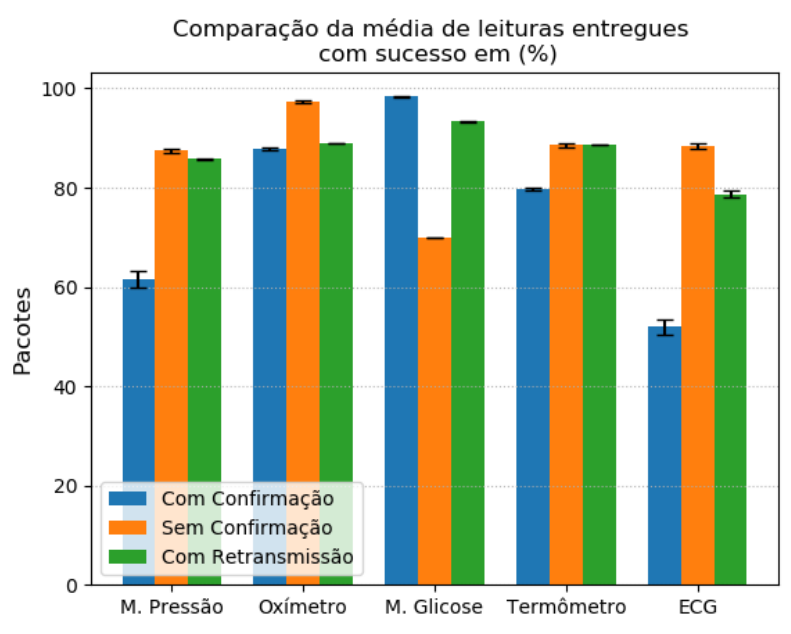

Figura 6. 0 eixo $y$ representa a percentagem de leituras entregues e o eixo $x$ os agentes. Cada linha do gráfico representa um modo de operação.

No modo de operação com confirmação, Figura 6, apenas o oxímetro e medidor de glicose entregaram mais de $85 \%$ das leituras. Devido o modo de operação sem confirmação não utilizar nenhum mecanismo de entrega confiável e nenhum timeout durante a transmissão, obteve um bom desempenho em quase todos os dispositivos.

Podemos observar que existe um trade-off quando 200 milissegundos é utilizado como timeout. Na Figura 7, é possível ver que o maior número de retransmissões ocorreu quando 200 milissegundos foi utilizado. Isso acontece em razão do gerente não enviar uma resposta dentro do tempo limite, fazendo com que os agentes reenviem o pacote por falta de ACK. O gráfico apresentado possui apenas valores do cenário onde maxNumOfRetransmition é igual a 6 , pois, a partir da sétima retransmissão, os valores tendem a ficar próximos de zero, como pode ser visto na Figura 7.

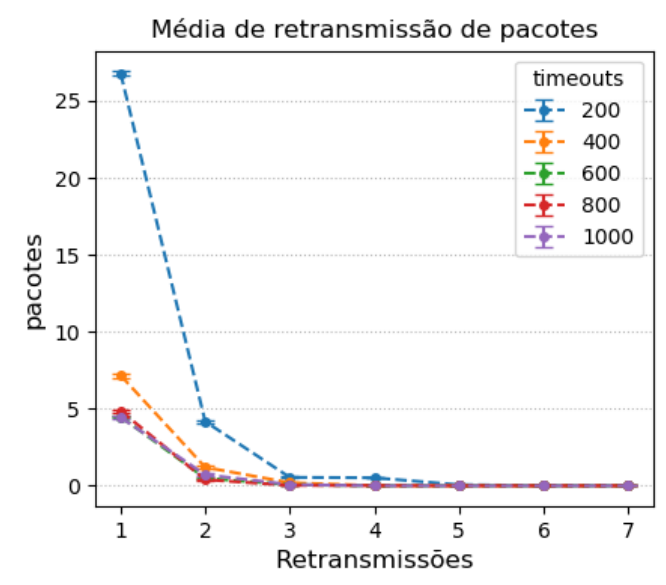

Figura 7. Retransmissões de pacotes utilizando timeouts diferentes.

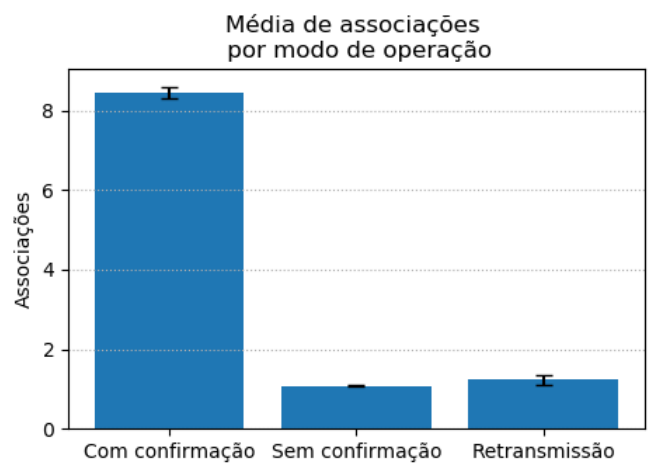

Figura 8. Média de associações feitas por agente, para 0 cenário com maxNumOfRetransmition $=6$ e timeOutToRetransmitPacket $=600 \mathrm{~ms}$.

Pode-se ver a redução no número de associações feitas por cada agente, entre o modo de operação com confirmação e o modo proposto no artigo na Figura 8. Em média, 
no modo de retransmissão, cada agente precisou estabelecer menos de duas associações para concluir a transmissão de leituras, enquanto o modo com confirmação usou em média mais de oito associações em cada transmissão. Isso representa uma economia de até 32 pacotes de controles, e vários timeouts evitados.

Na Figura 9, nota-se que a maioria dos pacotes possuem uma latência média de 30 milissegundos. O tempo é calculado desde a criação do pacote, na camada de aplicação do agente, até o recebimento do mesmo na camada de aplicação do gerente. Os valores mostrados são referentes ao cenário onde maxNumOfRetransmition é igual a 6 e timeOutToRetransmitPacket é igual a $200 \mathrm{~ms}$.

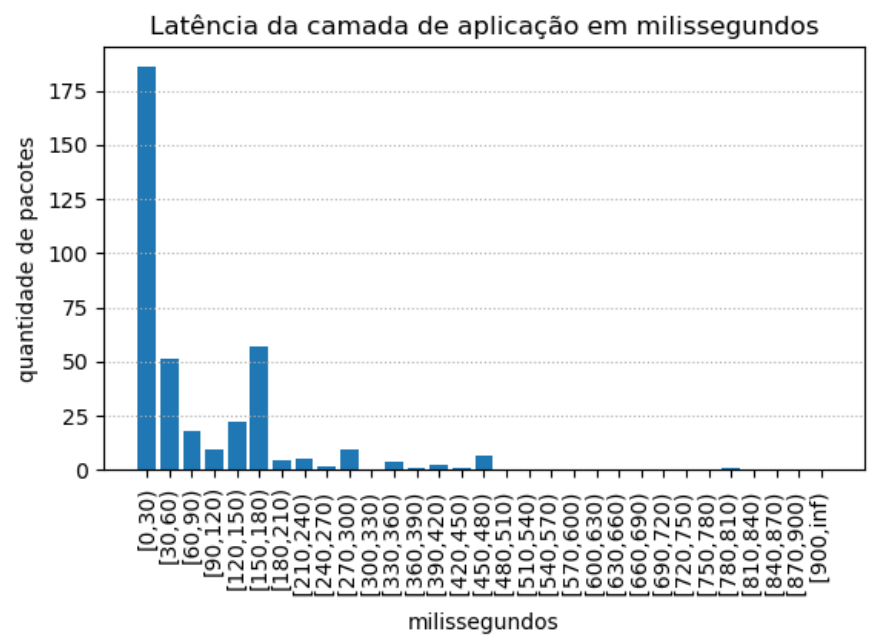

Figura 9. Latência média da camada de aplicação dos agentes, para o cenário com maxNumOfRetransmition $=6$ e timeOutToRetransmitPacket $=200 \mathrm{~ms}$.

Como visto nos resultados apresentados, o modo sem confirmação obteve uma das melhores performances, pois não possui nenhum tipo de mecanismo de entrega confiável e nem confirmação de entrega. O modo de retransmissão, alcançou um bom desempenho mesmo perdendo às vezes para o modo sem confirmação. Este modo entregou cerca de $15 \%$ mais pacotes de leituras e economizou $20.5 \%$ pacotes de controle, quando comparado ao modo com confirmação. Este último, infelizmente, demanda muito tempo e associações, o que resulta num delay alto e poucos pacotes entregues com sucesso.

\section{Conclusão}

Neste artigo, foi apresentada uma extensão ao protocolo X73-PHD que pode ser testado no Simulador Castalia e, adaptou dispositivos X73-PHD para serem empregados em cenários WBAN. Como ilustrado nos resultados, esta proposta melhora o desempenho dos PHDs, ao mesmo tempo que oferece um mecanismo de entrega confiável.

Como trabalhos futuros, será desenvolvida a função que permite ao usuário especificar, no gerente, a quantidade de dados que deve ser transmitida por cada agente. Os códigos da Aplicação do Castalia e da biblioteca Antidote modificada, podem ser encontrados nos endereços: https://github.com/conqlima/Antidote e https: //github.com/conqlima/11073PhdApplication. 


\section{Referências}

Boulis, A. and Tselishchev, Y. (2010). Contention vs. polling: a study in body area networks mac design. In Proceedings of the fifth international conference on body area networks, pages 98-104. ACM.

Boulis, Athanassios (2013). Castalia: A simulator for Wireless Sensor Networks and Body Area Networks (User's manual). https://github.com/boulis / Castalia. Online; accessed 7 January 2019.

Ferreira, V., Balbi, H., Seixas, F., Albuquerque, C., and Muchaluat-Saade, D. (2017). Wireless body area networks: An overview. XXXV Brazilian Communications and Signal Processing Symposium.

Goldberger, A., Amaral, L., Glass, L., Havlin, S., M. Hausdorg, J., Ivanov, P., G. Mark, R., E. Mietus, J., B. Moody, G., Peng, C.-K., Stanley, H., and Physiobank, P. (2000). Components of a new research resource for complex physiologic signals. PhysioNet, 101.

Gomes, Y. F., Santos, D. F., Almeida, H. O., and Perkusich, A. (2015). Integrating mqtt and iso/ieee 11073 for health information sharing in the internet of things. In Consumer Electronics (ICCE), 2015 IEEE International Conference on, pages 200-201. IEEE.

ISO/IEEE (2004). Ieee standard for health informatics - point-of-care medical device communication - part 20101: Application profile - base standard. ISO/IEEE 1107320101:2004(E), pages 01-04.

ISO/IEEE (2012a). Health informatics - personal health device communication part 00103: Overview. IEEE Std 11073-00103-2012, pages 1-80.

ISO/IEEE (2012b). Ieee standard for local and metropolitan area networks - part 15.6: Wireless body area networks. IEEE Std 802.15.6-2012, pages 1-271.

Jianchu, Y. and Steve, W. (2005). Applying the ISO/IEEE 11073 standards to wearable home health monitoring systems. Journal of Clinical Monitoring and Computing, 19(6):427-436.

Korsanov, I. N., S.M, K., D.A, R., V.V, F., and M.A, S. (2013). On demand plugins for X73-PHD manager. International Journal of Advanced Trends in Computer Science and Engineering, 2(5):35-40.

Martins, A. F., Santos, D. F., Perkusich, A., and Almeida, H. O. (2014). Ieee 11073 and connected health: Preparing personal health devices for the internet. In Consumer Electronics (ICCE), 2014 IEEE International Conference on, pages 274-275. IEEE.

Movassaghi, S., Abolhasan, M., Lipman, J., Smith, D., and Jamalipour, A. (2014). Wireless body area networks: A survey. IEEE Communications Surveys Tutorials, 16(3):1658-1686.

Santos, D. F., Bublitz, F. M., Almeida, H. O., and Perkusich, A. (2014). Integrating ieee 11073 and constrained application protocol for personal health devices. In Proceedings of the 29th Annual ACM Symposium on Applied Computing, pages 466-467. ACM.

Signove (2011). Antidote: Program Guide, documentation for developers of applications based on Antidote IEEE 11073 library. http: / / oss.signove.com/images / c/c7/AntidoteProgramGuide.pdf. Online; accessed 7 January 2019. 